\title{
Diagnostic accuracy of different CMR techniques in the evaluation of ischemic cardiomyopathy
}

\author{
Eugene Won ${ }^{1 *}$, Angela Tong ${ }^{4}$, Ruth P Lim³ ${ }^{3}$,Tatyana Danilov², Leon Axel', Monvadi B Srichai ${ }^{1}$ \\ From 16th Annual SCMR Scientific Sessions \\ San Francisco, CA, USA. 31 January - 3 February 2013
}

\section{Background}

In patients newly diagnosed with heart failure, coronary angiography is the current gold standard in differentiating underlying coronary artery disease and non-ischemic cardiomyopathy. However, CMR is emerging as an attractive alternative that can provide substantially more diagnostic information with its multiple sequences, while also demonstrating a superior safety profile. In this study, we evaluated the diagnostic ability of late gadolinium enhancement (LGE), cine, and first pass perfusion (FPP) in diagnosing ischemic cardiomyopathy compared to coronary angiography (CA).

\section{Methods}

Retrospective chart review identified 109 consecutive patients (68\% male, average age 58 (range 23-84 years), average ejection fraction $30 \%$ (range 10-50\%) referred for CMR evaluation of new onset cardiomyopathy with CA performed within 2 weeks of CMR. Both imaging tests were performed within two months of clinical heart failure diagnosis. Patients were examined with cine, FPP, and
LGE sequences as part of the CMR protocol. Ischemic cardiomyopathy was defined based on the standardized criteria proposed by Felker et al. - stenosis of 70\% or greater in the left main vessel or proximal LAD or at least two epicardial coronary arteries as identified on CA. Cine images were evaluated for regional and global wall motion abnormalities (hypokinesia/dyskinesia). FPP images were evaluated for presence of regional hypoperfusion. LGE images were evaluated for subendocardial and/or transmural enhancement consistent with an ischemic pattern in a coronary artery distribution. These sequences were then analyzed for their predictive value in the diagnosis of ischemic cardiomyopathy.

\section{Results}

CA identified 66 patients (60.6\%) with ischemic cardiomyopathy. Diagnostic accuracy, sensitivity, specificity, PPV, NPV and ROC analysis of individual and combinations of techniques for diagnosis of ischemic cardiomyopathy are presented in Table 1. Using information from all three techniques resulted in near perfect sensitivity at

Table 1 Diagnostic accuracy, sensitivity, specificity, positive predictive value, and negative predictive value for the combinations of CMR techniques in diagnosing ischemic cardiomyopathy compared to CA.

\begin{tabular}{|c|c|c|c|c|c|c|}
\hline Combination of CMR sequences & Sensitivity & Specificity & Positive predictive value & Negative predictive value & diagnostic accuracy & ROC \\
\hline CINE & $61 \%$ & $60 \%$ & $70 \%$ & $50 \%$ & $61 \%$ & 0.61 \\
\hline FPP & $83 \%$ & $49 \%$ & $72 \%$ & $65 \%$ & $67 \%$ & 0.64 \\
\hline LGE & $89 \%$ & $63 \%$ & $79 \%$ & $79 \%$ & $79 \%$ & 0.76 \\
\hline CINE, FPP & $94 \%$ & $35 \%$ & $69 \%$ & $79 \%$ & $71 \%$ & 0.64 \\
\hline LGE, CINE & $94 \%$ & $44 \%$ & $72 \%$ & $83 \%$ & $74 \%$ & 0.69 \\
\hline LGE, FPP & $97 \%$ & $42 \%$ & $72 \%$ & $90 \%$ & $75 \%$ & 0.69 \\
\hline CINE, FPP, LGE & $97 \%$ & $30 \%$ & $68 \%$ & $89 \%$ & $71 \%$ & 0.64 \\
\hline
\end{tabular}

$\mathrm{CINE}=$ regional wall motion abnormality on cine, FPP = regional hypoperfusion on resting first pass perfusion, LGE $=$ ischemic pattern on late gadolinium enhancement

${ }^{1}$ Medicine, Radiology, NYU School of Medicine, New York, NY, USA

Full list of author information is available at the end of the article 
the expense of specificity. McNemar test comparing diagnostic accuracies of tests showed that LGE-CMR alone was significantly better than the other two sequences alone.

\section{Conclusions}

Ischemic pattern of LGE alone demonstrates the best diagnostic performance for diagnosis of ischemic cardiomyopathy compared to other individual and combination CMR techniques. A combination of techniques may be needed to improve the diagnostic sensitivity and negative predictive value in order to ensure identification of patients who may benefit from revascularization.

\section{Funding}

None.

\section{Author details}

${ }^{1}$ Medicine, Radiology, NYU School of Medicine, New York, NY, USA.

${ }^{2}$ Medicine, Beth Israel Hospital, New York, NY, USA. ${ }^{3}$ Radiology, Austin Health,

Melbourne, VIC, Australia. ${ }^{4}$ New York Medical College, Valhalla, NY, USA.

Published: 30 January 2013

doi:10.1186/1532-429X-15-S1-E71

Cite this article as: Won et al: Diagnostic accuracy of different CMR

techniques in the evaluation of ischemic cardiomyopathy. Journal of Cardiovascular Magnetic Resonance 2013 15(Suppl 1):E71.
Submit your next manuscript to BioMed Central and take full advantage of:

- Convenient online submission

- Thorough peer review

- No space constraints or color figure charges

- Immediate publication on acceptance

- Inclusion in PubMed, CAS, Scopus and Google Scholar

- Research which is freely available for redistribution

Submit your manuscript at www.biomedcentral.com/submit
C Biomed Central 